\section{Doctor meets patient}

Therapeutics: From the Primitives to the 20th Century, with an AppendixHistory of Dietetics. By Erwin $\mathbf{H}$. Ackerknecht. Pp. $\mathrm{x}+194$. (Hafner, Macmillan: New York; Collier Macmillan: London, January 1974.) £6.25.

Medical historians have all too often shown greater interest in what doctors have written than in how they have treated their patients. They have studied medical ideas and theories rather than the ways these ideas have been applied. Certainly, theory fascinates more than praxis. Yet for the patient at least, praxis is the key medical activity, patients being far more concerned with what their physicians can do than with what they know.

Therapeutics is the point where doctor and patient meet. Nevertheless, such has been the neglect of this subject by medical historians that the last general history of therapeutics was published in 1877-a fact which makes Professor Ackerknecht's lucid and stimulating volume all the more welcome. First published in German in 1970, it has now been translated by the author, formerly Director of the Institute of Medical History at the University of Zürich. The book charts the therapeutic programmes and rationales of medicine from the Egyptians to the twentieth century. Ackerknecht's method involves analysis of the therapeutic writings of major medical figures in each particular age: the Hippocratics, Galen, Paracelsus, Sydenham, Laennec, Ehrlich, and so on. In addition, he refers frequently to the works of less well known doctors whose activities and thoughts were equally significant in defining the medical texture of each of his historical portraits. The result is a monograph which is crammed with facts and brimming with ideas.

It is difficult to summarise a book which is so wide ranging. Nevertheless, two recurrent themes should be mentioned. First, as Ackerknecht repeatedly demonstrates, medical theories have been far more flexible than medical therapeutics. Bloodletting, for instance, has been employed in many different settings. The primitives used it. The Greeks recommended it, as did the Indians. Bloodletting was practised in the Middle Ages, the Renaissance and the Enlightenment. In fact only in the middle of the nineteenth century did physicians begin to look critically at this traditional form of therapy. The therapy itself remained essentially unchanged, yet the rationale for this therapeutic procedure could be different for each particular age or culture setting. The same may be said for many other traditional therapies, such as purgatives, cathartics and emetics.
The second historical insight which Ackerknecht brings to bear on his subject concerns the nature of medical experience. In therapeutics, each doctor should be able to form independent evaluations of the effectiveness of his armamentarium. Unfortunately, experience often being no more than 'pseudo-experience', doctors have found that any particular therapeutic regimen yields precisely the results they expected it to. Patients rarely disappoint their physicians if they can help it.

Professor Ackerknecht explores the historical ramifications of these and many other themes in this splendid little book. A full appreciation of it requires some prior acquaintance with the general features of the history of medicine. This can be pleasantly acquired through Ackerknecht's Short History of Medicine, published in 1955 but still the best short introduction to the subject available. The remarkable range of his interests may be seen from some of his other contributions to the history of medicine: a biography of Rudolf Virchow, a study of French medicine in the first half of the nineteenth century, a monograph on malaria in the Upper Mississippi Valley, a short history of psychiatry, and a series of classic papers on primitive medicine. These works mark Erwin Ackerknecht as a true cosmopolitan and one of the outstanding medical historians of our time.

W. F. BYNUM

\section{Rejecting behaviourism}

The Psychology of Consciousness. By Robert E. Ornstein. Pp. xxi+247. (Freeman: San Francisco, 1972.) $\$ 3.50$.

THIs book represents a current swing away from behaviourist psychologies, in which consciousness is ignored or even denied. It criticises Western science, as being over-limited to sequential processes of reasoning at the cost of immediate intuitive understanding, associated with the Eastern approach to studying mind represented by the Chinese I Ching and the meditative and rhythmic exercises of yoga. Dr Ornstein tries to relate East and West, with the neurological notion that (in right handed people) the right cerebral hemisphere serves the artist and dreamer, while the left hemisphere serves sequential logical thinking and language. For this he refers to the important 'split brain' experiments of Roger Sperry, in which the fibres of the corpus callosum, normally joining the hemispheres, are sectioned in serious cases of epilepsy.

Ornstein suggests that the righthemisphere Eastern culture and the lefthemisphere Western culture can be combined-to make better use of the brain as a whole. Is this a neurological alliance of C. P. Snow's Two Cultures?

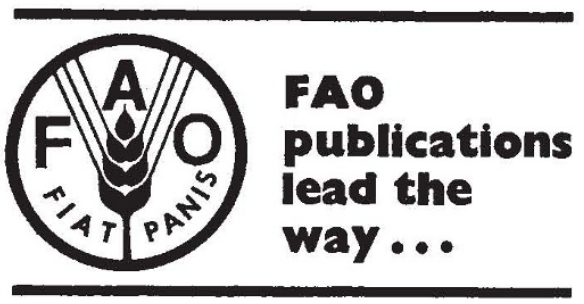

Eighteen distinguished scientists have contributed to this pioneer book of detailed description and explanation, the definitive volume on:

\section{The Husbandry \& Health of the Domestic Buffalo}

Edited by W. Ross Cockrill

(English edition only)

An authoritative compendium of existing knowledge of the water buffalo and its superior ability for development in meat production, milk yield, and work output. (223 illustrations, 993 pages)

Price: Clothbound $\mathbf{\$ 8 . 0 0}$ (postage extra)

AVAILABLE SEPTEMBER 1974

(Please place your order now)

Please send your order(s) to:

Her Majesty's Stationery Office

PM1A(88), Room C46

Atlantic House

Holborn Viaduct

London ECIP IBN

PLEASE USE BLOCK CAPITALS

TICK APPROPRIATE BOX

I wish to order ........... copy(ies) of

The Husbandry and Health of the Domestic

Buffalo at $£ 8.00$ each (postage extra)

Please invoice my HMSO Account

No.......................

Payment of $£ \ldots \ldots \ldots$ is enclosed

Postpaid surface mail

Airmail at my expense

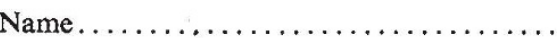

Address

Food and Agriculture Organisation of the United Nations

Distribution and Sales Section Via della Terme di Caracalla ROME - Italy 